\title{
Biosensing of hepatitis B antigen with poly(acrylic acid) hydrogel immobilized with antigens and antibodies
}

\begin{abstract}
Hydrogel based on poly(acrylic acid) (PAAc) polymers was successfully fabricated as the biosensor for detecting hepatitis B core antigen ( $\mathrm{HBcAg})$. Specifically, the pendant $\mathrm{HBcAg}$ and the anti-hepatitis B core antigen (anti-HBc) antibody were first immobilized on the PAAc, which were then covalently cross-linked via radical polymerization to form the HBcAg-sensitive (HBPAAc) hydrogel. The non-covalent affinity binding between the immobilized $\mathrm{HBcAg}$ and anti-HBc would be disrupted by the presence of free $\mathrm{HBcAg}$ in the HBPAAc hydrogel. The competitive binding of free $\mathrm{HBcAg}$ on the immobilized anti-HBc triggered the swelling of HBPAAc hydrogel. The equilibrium swelling ratio and the oscillatory swelling-deswelling kinetics of the HBPAAc hydrogel in response to protein concentration were studied. The swelling ratio of HBPAAc hydrogel increased along with an increase in $\mathrm{HBcAg}$ concentration until equilibrium was achieved at $4 \mathrm{mg} / \mathrm{mL} \mathrm{HBcAg}$. The HBPAAc hydrogel did not exhibit swelling/deswelling behavior when interacted with the negative control, i.e., bovine serum albumin (BSA). Based on the result of oscillatory swelling-deswelling of HBPAAc hydrogel, the minimum duration for $\mathrm{HBcAg}$ detection by the HBPAAc hydrogel was 20 min, whereas the regeneration of HBPAAc hydrogel took about $60 \mathrm{~min}$. These results confirmed the reversibility and the reusability of this $\mathrm{HBcAg}-$ sensing HBPAAc hydrogel. Besides, the hydrogel demonstrated zero cross-reactivity to the hepatitis B surface antigen, a common serological marker for hepatitis B patients. The high sensitivity of HBPAAc hydrogel to the $\mathrm{HBcAg}$ was successfully demonstrated with quartz crystal microgravimetry. The magnitude of resonance frequency was inversely correlated with the swelling of HBPAAc hydrogel, which is governed by the concentration of analyte HBcAg. The application of HBPAAc hydrogel as a biosensor component in the detection and surveillance of hepatitis B holds great promises.
\end{abstract}

Keyword: Hydrogel; Acrylic acid; Hepatitis B core antigen (HBcAg); Bioresponsive; Swelling; Biosensor 